\title{
El Auto o Comedia de la Soberana Virgen de Guadalupe y de sus milagros y grandezas de España: descripción y estudio bibliográfico de dos testimonios de una obra atribuida a Cervantes \\ Auto or Comedia de la Soberana Virgen de Guadalupe y de sus milagros y grandezas de España: Description and Bibliographic Study of Two Testimonies of a Drama Attributed to Cervantes
}

\section{Fructuoso Atencia Requena}

Universidad Complutense de Madrid ESPAÑA

fatencia@ucm.es

[Hipogrifo, (issn: 2328-1308), 8.1, 2020, pp. 345-358]

Recibido: 06-08-2019 / Aceptado: 25-09-2019

DOI: http://dx.doi.org/10.13035/H.2020.08.01.24

Resumen. El Auto o Comedia de la Soberana Virgen de Guadalupe y de sus milagros y grandezas de España es una obra cuyo interés principal ha girado siempre en torno a su hipotética autoría cervantina. El siguiente artículo lleva a cabo un repaso por toda la atención bibliográfica que dicha atribución ha suscitado, y elabora en profundidad, desde la disciplina de la bibliografía material, una descripción tipobibliográfica de la edición impresa de 1617 y una descripción codicológica del manuscrito, para intentar establecer la relación de filiación existente entre los dos únicos testimonios áureos conservados del texto. 
Palabras clave. Comedia de la Soberana Virgen de Guadalupe y de sus milagros y grandezas de España; Cervantes; testimonios; filiación; descripción tipobibliográfica y codicológica.

Abstract. Auto or Comedia de la Soberana Virgen de Guadalupe y de sus milagros y grandezas de España is a drama which main interest always revolves around its hypothetical Cervantine authorship. The following paper carries out all bibliographic attention that said attribution has generated, and it elaborates deeply, from the discipline of the Material Bibliography, a bibliographic-type description of the 1617 print edition and a codicological description of the manuscript, to try to establish the relationship of affiliation between the only two preserved testimonies of the text.

Keywords. Comedia de la Soberana Virgen de Guadalupe y de sus milagros y grandezas de España; Cervantes; Testimonies; Filiation; Bibliographic-type and codicological description.

\section{ESTADO DE LA CUESTIÓN: EL AUTO O COMEDIA DE LA SOBERANA VIRGEN DE GUADALUPE Y DE SUS MILAGROS Y GRANDEZAS DE ESPAÑA, ¿UNA OBRA CERVANTINA?}

La atención bibliográfica que ha suscitado la obra recogida en los testimonios que pretendemos estudiar, surge de la hipótesis que algunos estudiosos han planteado sobre la autoría de la misma. Como enuncia Eisenberg, «se ha sugerido que sea de Cervantes una comedia llamada La soberana Virgen de Guadalupe y sus milagros y grandezas de España, aunque hoy en día no tiene mucho apoyo»' '. Dicha obra pertenece a esa serie de atribuciones dudosas que aún rondan el corpus de Cervantes y que han abordado importantes cervantistas como Avalle-Arce ${ }^{2}$, Eisenberg $^{3}$ o Montero Reguera ${ }^{4}$. El primero en atribuir esta comedia al escritor de la Numancia fue Juan Colón y Colón ${ }^{5}$. Esta idea es defendida por Asensio en su edición de la Comedia de la Soberana Virgen de Guadalupe y sus milagros y grandezas de España de 1868; una justificación que, aun refrendada por Menéndez Pelayo6, según Ortega Morejón ${ }^{7}$ y posteriormente Montero Reguera, utiliza «argumentos ya lo anticipo- muy ligeros y superficiales para la filiación cervantina del texto»8. Crémoux, por su parte, no encuentra tan descabellada la hipótesis de Asensio, y pronuncia a este respecto:

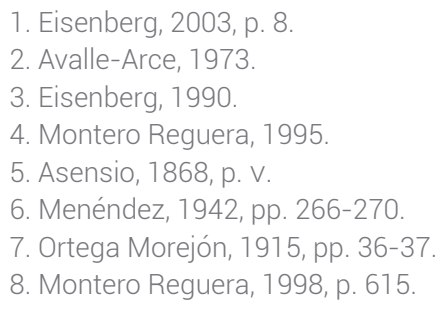


Es interesante apuntar que algunos de los documentos manejados por Asensio en su intento de demostrar la autoría de Cervantes dejan entrever un posible vínculo entre el mundo de los cautivos y el autor de la obra; Asensio incluso emite la hipótesis de que la obra haya sido compuesta en cautiverio en Argel, y representada delante de un público de cautivos. Esto podría explicar tanto la elección del tema, siendo la Virgen de Guadalupe tan popular entre los cautivos, como el tono virulento de denuncia de los moros ${ }^{9}$.

Si bien es cierto que los argumentos en los que se basa Asensio pueden parecer algo triviales, no lo es menos que estos se sustentan en textos del propio Cervantes. El primer argumento de que se sirve para demostrar que esta obra es cervantina es que el escritor, tal como muestra en Los trabajos de Persiles y Sigismunda, era un ferviente devoto de la Virgen de Guadalupe ${ }^{10}$.

Esta justificación, válida pero muy general, es seguida de otro razonamiento que podría resultarnos más interesante, conociendo el gusto de Cervantes por los juegos literarios. Asensio se fija en los nombres de dos de los personajes de la comedia, y encuentra que tienen correspondencia con los nombres de personajes de otro par de obras cervantinas que tratan el tema del cautiverio de Argel:

En la Comedia se encuentra la mujer del pastor de Cáceres, que tiene por nombre Aurelia, y nos hace recordar que Aurelio se nombra el cautivo de El trato de Argel; y el niño hijo de aquella pastora en quien la Virgen hace el milagro, es llamado Francisquito, como llamó Cervantes al niño mártir de sus Baños de Argel ${ }^{11}$.

Sin embargo, mientras que Ortega Morejón defiende que «no debe tenerse esa circunstancia como testimonio de que una y otras obras nacieran del mismo numen, pues no acostumbra Cervantes a dar a las creaciones de su genio nombres iguales» ${ }^{12}$, para Montero Reguera las mencionadas por Asensio son «ciertas coincidencias, muy leves», y los argumentos «no tienen la más mínima solidez» ${ }^{13}$. La última de las pruebas que Asensio ofrece para justificar su hipótesis es, en sus propias palabras, «la del estilo», apoyada también por Hartzenbusch, quien afirma que «la comedia no desdice del estilo dramático de nuestro gran escritor» ${ }^{14}$. A este respecto, Montero Reguera opina de nuevo y enuncia que «el juicio de Hartzenbusch tampoco mejora ni perfila la hipótesis de Asensio» ${ }^{15}$.

Lo cierto es que la cuestión de si esta obra es o no de Cervantes ha hecho correr ríos de tinta y ha enfrentado a numerosos estudiosos desde el siglo XIX hasta el $x x$ que, o bien han apoyado la atribución con más o menos fundamento pero sin la seguridad necesaria, o bien han rechazado los argumentos de sus contrarios. Junto con Ortega Morejón, que mediante numerosas pruebas aduce que la obra

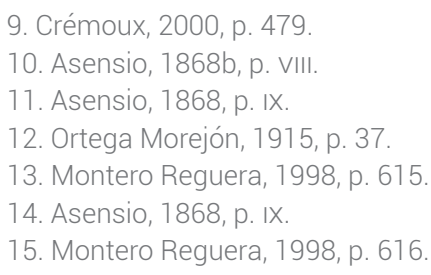


no es cervantina, el caso más llamativo es el de Astrana Marín, quien arremete contra todos y cada uno de los estudiosos que han defendido que la Comedia de la soberana Virgen de Guadalupe es hija de Cervantes ${ }^{16}$. Tal repercusión debieron alcanzar sus palabras a este respecto que desde la publicación de su Vida ejemplar y heroica de Miguel de Cervantes Saavedra (mediados del siglo XX) «las referencias a esta obra son mínimas y quedan reducidas prácticamente a breves entradas, que no aclaran nada» ${ }^{17}$.

Junto a estas encontramos también posiciones intermedias, como las defendidas por Sánchez Arjona o el mismo Montero Reguera. El primero demuestra documentalmente que la obra fue escrita y representada en 1594 en Sevilla con motivo de unas justas poéticas que se celebraron para la festividad del Corpus. Aclarado este punto, da esta opinión acerca de la posible autoría cervantina:

En cuanto que Cervantes sea el autor del auto de Nuestra Señora de Guadalupe, creemos que bien pudo serlo sin necesidad de recurrir a esta multitud de circunstancias que imagina el Sr. Asensio, toda vez que en este año Cervantes vivía en Sevilla y pudo concurrir a esta especie de certamen, y acaso el no haber sido premiado le hiciera ocultar su nombre ${ }^{18}$.

Por su parte, Montero Reguera concluye que, dado que los argumentos a favor de que la obra sea cervantina no son de gran peso, esta debe ser descartada del corpus del genial escritor. Sin embargo, rompe una lanza a favor de la hipótesis de Sánchez Arjona, y deja abierta una puerta a la posibilidad de que el Auto de Nuestra Señora de Guadalupe sea de Cervantes:

Es posible, pues Cervantes estaba allí, que nuestro escritor fuera el autor de ese auto no premiado en 1594. Pero en tanto en cuanto no se localice algún documento que así lo pruebe fehacientemente, la obra permanecerá anónima ${ }^{19}$.

\section{TRANSMISIÓN DE LA OBRA}

Los testimonios del Auto o Comedia de la Virgen de Guadalupe que nos han llegado son pocos. Anteriores al siglo xx solo conservamos dos ediciones impresas de la obra: una del año 1617, publicada en Sevilla por Gómez de Pastrana, y otra ya del siglo XIX, concretamente de 1868, publicada también en Sevilla, que recoge el texto de la anterior. A estos dos testimonios impresos hemos de añadir un manuscrito incompleto de la obra que se conserva en la Biblioteca Nacional bajo la signatura Ms.15157. A continuación realizaremos una descripción tipobibliográfica de la edición de 1617, y una descripción codicológica del manuscrito.

16. Astrana Marín, 1958, p. 760.

17. Montero Reguera, 1998, p. 617.

18. Sánchez, 1898, «Auto de La Virgen de Guadalupe, atribuido a Cervantes», p. 90.

19. Montero Reguera, 1998, p. 617. 


\title{
2.1. Las ediciones impresas
}

El ejemplar de la primera edición impresa que conservamos, el de 1617, nos deja unos datos interesantes acerca de la licencia de impresión y de las personas que la solicitaron. La licencia fue otorgada en Madrid en 1598, y la tasa es de 1608. Sin embargo, pese a ser esta la primera edición de que disponemos, sabemos por documentos de la época que hubo otras:

\begin{abstract}
El Catálogo de la biblioteca de Salvá (vol. I, pp. 362b-363a) señala la existencia de una edición de 1615 -que, pese a mis esfuerzos, no he podido encontrar ejemplar-, también impresa en Sevilla por Bartolomé Gómez, y de iguales características - señala Salvá - que la de 1617. También señala el mismo catálogo, lo cual es más importante, la existencia de una edición de 1605 -ejemplar de la propiedad de Salvá cuyo paradero hoy se desconoce-, donde la obra recibe el título de Auto de la soberana Virgen de Guadalupe ${ }^{20}$.
\end{abstract}

Domínguez Guzmán, en su obra La imprenta en Sevilla en el siglo XVII, recoge también, además de la de 1617, dos ediciones impresas más de la obra, una de 1605 que lleva el título de Auto de la Soberana Virgen de Guadalupe, y sus milagros, y grandezas de España, y otra de 1615, tal como enunciara Salvá21.

\section{DESCRIPCIÓN TIPOBIBLIOGRÁFICA DE LA EDICIÓN DE 1617}

\section{Noticia bibliográfica sintética:}

Comedia de la Soberana Virgen de Guadalupe y sus milagros y grandezas de España, Sevilla, por Bartolomé Gómez de Pastrana, a la Cárcel Real, 1617.

\section{Características técnicas: colación y notas complementarias:}

\section{4. ${ }^{0}-A^{8} .-8$ h.- L. red.- 2 pliegos}

Inic. tip.- Texto de la loa en verso a 2 cols., texto de la comedia en verso a 1 y 2 cols., licencia y tasa a línea tirada.- Con reclamos.

\section{Transcripción facsimilar y relación de contenido:}

[A1] r: Portada:

COMEDIA DE LA I SOBERANA VIRGEN DE GVA- I dalupe, y fus Milagros, y Grandezas I de Elpaña. I 9 SON INTERLOCVTORES. I [1 . ${ }^{a}$ col.] 9 Benhalamar Moro.l 9 Abenamar Moro. 19 Aliatarfe Moro. 19 Cegrino Moro. Alarico Godo. 19 Silibuto Godo.

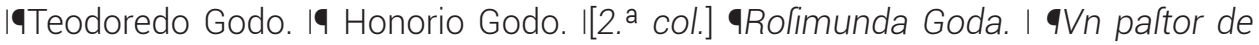


Caceres. I Aurelia fu muger. 19 Francifquito fu hijo. (res I Dos Ciudadanos de Cace I El Cura de Caceres. 19 Marcelo paltor. I Nra Señora de Guadalupe. I [Grab. xil. que representa tres figuras, la Virgen con el Niño dentro de un medallón ovalado y una figura a cada lado, un caballero a la izquierda y una dama a la derecha. La escena que conforman las tres figuras está enmarcada por una orla abierta en su parte superior formada por tres piezas xilográficas con decoración vegetal.] I Con licencia, imprefla en Sevilla, Por Bartolome Gomez de I Paftrana, à la Carcel Real. I Año de 1617.I

[A1] v: Loa: LOA.I Texto de la loa: (col. 7): E² Poderolo Alexādro, I coronado en Macedonia I gran Emperador del mundo, I y grāde moltruo en hiftorias I Porque el Retrato del Templo I no borrallen gentes tolcas... (Al fin, col. 2, lín. xx): hazelde mudo de lengua, I por ef́pacio de dos horas.

A2 v-A8 r: Texto de lacomedia: Tocan de dentro arebato, y falen los I quatro Moros con las efpadas defnudas, y Aliatarfe I con la Vandera. I [Inicio del diálogo teatral, a una columna]: Benhalamar. I V²aliente affalto. Aliatarfe. Braua efcaramuça I A pefar de las armas del Chrítiano, I Cegrimo. Ya el valiente Épañol las armas cruza, I Y fiente en fu cerviz el pie Africano: I Aliatarfe. Planta en lo alto effe Pendon de Muçal Del humillado Alcayde Seuillano, I Valiente Benhalamar, cuya gloria ISerà cierta feñal de la Vitoria... (Al fin, col. 2, lín. xx):Paft. Todo el pueblo le acompaña, I y da fin la alegre Hiftoria I de deuocion y memoria I de las Grandezas de Elpaña.I [Centrado y entre dos adornos vegetales]: LAVS DEO. I A8 v: Licencia, dada en Madrid a 10 de agosto de 1598: LICENCIA. I Y²o Gonçalo de la Vega, Elcriuano de Camara del rey I nueltro leñor, doy fee,...।

A8 v: Tasa, a cinco blancas el pliego, dada en Madrid a 12 de octubre de 1608: TASSA. I Y ${ }^{2}$ o Francilco Martinez, Elcriuano de Camara de fu Mageftad, y I vno de los que en el fu... I Francilco Martinez.

ASENSIO, José María, «Teatro español anterior a Lope de Vega. ¿Miguel de Cervantes?», en Comedia de la soberana Virgen de Guadalupe y de sus milagros y grandezas de España, 1868.-CCPBE. n. 000039808-X.- MENÉNDEZ PELAYO, Marcelino, «Cervantes considerado como poeta», en Estudios y discursos de crítica histórica y literaria. Estudios generales. Edad Media influencias semíticas. Cervantismo, 1942.- MONTERO REGUERA, José, «Hacia una revisión de las atribuciones teatrales cervantinas: el auto de la Virgen de Guadalupe», en Actas del Tercer Congreso Internacional de la Asociación de Cervantistas, 1998- ORTEGA MOREJÓN, José María, «Primeros apuntes. La Comedia de la Soberana Virgen de Guadalupe y sus milagros y grandezas de España», en Apuntes para dos obras relacionadas con Cervantes con algunos documentos inéditos, 1915. - PALAU y DULCET, III, 53.984. - SÁNCHEZ ARJONA, José, «Auto de La Virgen de Guadalupe, atribuido a Cervantes», en Noticias referentes a los anales del teatro en Sevilla desde Lope de Rueda hasta fines del siglo XVII, 1898. - SIMÓN DÍAZ, BLH, VIII, núm. 1609.

Ejemplares cotejados: MADRID, Biblioteca Nacional, T/10886 (olim. I-5) [Ex libris de la «Librería del licenciado D. Cayetano Alberto de la Barrera» adherido a la contratapa anterior]*. 


\section{Notas adicionales sobre particularidades de esta edición:}

Al parecer, es el único ejemplar conservado de esta edición. El impreso va foliado a mano, de letra posterior, que comienza con el número 59 y abarca hasta el 66, lo que indica que este ejemplar formó parte de un volumen facticio.

En lo que respecta a la disposición del texto22, el recto de la primera hoja es la portada, que incluye el título, los nombres de los personajes de la comedia dispuestos en dos columnas y una estampa de la Virgen, junto a dos figuras, enmarcada por una orla xilográfica de composición, abierta por arriba; bajo la estampa aparecen los datos de impresión (lugar, año y nombre del impresor). La «loa», que ocupa el vuelto de la primera hoja y el recto de la segunda, más un tercio de su vuelto, está a dos columnas. El comienzo de la comedia se encuentra a partir del segundo tercio del vuelto de la segunda hoja, y el texto se dispone ahora a una columna hasta la mitad del recto de la tercera hoja. Desde aquí y hasta el recto de la octava hoja, en cuyo final hallamos el Laus Deo, la disposición del texto es a dos columnas, sin duda para no excederse de los dos pliegos conjugados que conformaban muchas veces estas piezas sueltas. En el vuelto de la octava hoja encontramos la licencia y la tasa, en prosa, que ocupan cada una media cara.

La letra es redonda (curiosamente no hay nada en cursiva), y el pliego está impreso en tinta negra. La tipografía presenta unas características mínimas que podemos señalar. Las letras capitulares de la loa (E) el principio de la comedia (V) y la de la primera escena en la que sale Benhalamar (D) tienen la altura de dos líneas de escritura. Esta característica no vuelve a aparecer en el impreso hasta el vuelto de la última hoja, en el que las primeras letras de la licencia y la tasa (Y) también ocupan dos líneas de altura. En todas las hojas, exceptuando el recto de la primera -la portada - y el vuelto de la octava - la última- encontramos reclamos en el ángulo inferior derecho.

Los únicos elementos ornamentales son la ilustración de la portada y dos adornitos tipográficos en forma de hojas acorazonadas que encierran el Laus Deo. La encuadernación es moderna, en holandesa.

\subsection{EL MANUSCRITO}

El primer estudioso que reparó en este manuscrito, según sus propias palabras, fue Montero Reguera ${ }^{23}$. Él hace una breve descripción del manuscrito, y cita algunas diferencias de este con respecto a la edición de 1617. Su observación, acertada pero sucinta, se centra en ciertas variantes textuales relativas a las acotaciones y el listado de personajes. A continuación haremos una descripción más detallada del manuscrito y del volumen facticio en el que se encuentra, y analizaremos ciertos detalles que nos han llamado la atención y que nos parecen relevantes para inten-

22. Inserto aquí esta explicación de la disposición porque será relevante en su comparación con la disposición del manuscrito sobre la misma obra que se conserva.

23. Montero Reguera, 1998, p. 614. 
tar descifrar cuál es su naturaleza (autógrafo, apógrafo, copia de imprenta...) y qué relación puede mantener con la edición impresa que de la obra se conserva.

\section{DESCRIPCIÓN CODICOLÓGICA DEL MANUSCRITO 15157 DE LA BNE}

MADRID, Biblioteca Nacional. Ms. 15157.

Auto a Nuestra Señora del Pilar y Auto de la Virgen de Guadalupe y sus milagros y grandezas de España. [Título del catálogo de la BNE]: Autos sacramentales (manuscrito).

4. ${ }^{0}-11$ h., foliación a lápiz, papel, letra cursiva del siglo XVII. Texto en verso. En español. Encuadernación rígida posterior en holandesa.

s. XVII A; s. [XVII] B

Ms. facticio, papel: $216 \times 150 \mathrm{~mm} ; 11 \mathrm{~h}$.

\section{Sector A}

\section{[Auto de Nuestra Señora del Pilar]}

fol. 1r RUB.: Auto a Nuestra Señora del Pilar. De un yngenio.

TABLA: Personas. El hombre. La Devoción. El día... furia...

INC.: De una gruta sale el furor. Fur. Conducido de Luzbel / y mandado de su

fol. 9v EXP.:... la devoción de la fiesta / de la Virgen del Pilar.

RUB.: Año de 1695.

\section{Sector B}

[Auto de la Virgen de Guadalupe y sus milagros y grandezas de España]

fol. 10r RUB.: Auto famoso de nuestra señora de guadalupe y sus milagros; y grandezas de España.

TABLA: Figuras. Benalamar, moro. Abenamar, moro. Aliatarfe, moro...

INC.: Tocan dentro arrebate y salen los quatro moros con las espadas desnudas en las manos y aliatarfe con la bandera...

fol. 11 v. FTC... ... rodrigo mal fadado [...].

El manuscrito, perteneciente al fondo antiguo de la Biblioteca Nacional de España, forma parte de un volumen facticio que contiene dos manuscritos, el primero de ellos completo y otro del que solo se conserva un fragmento. El primero, que lleva por título Auto a Nuestra Señora del Pilar, es de 1695. El segundo no está datado, 
aunque por la letra podemos suponer que también es del siglo XVII. El manuscrito consta de un total de 11 hojas, y la foliación, moderna, aparece en el ángulo superior externo del recto de cada hoja, a lápiz, en números romanos y arábigos ${ }^{24}$. El texto del Auto de Nuestra Señora del Pilar está en verso y a dos columnas. Por su parte, la disposición del Auto de Nuestra Señora de Guadalupe, también en verso, es distinta, y está a una y dos columnas.

El soporte es papel, y el cuaderno del primer manuscrito es un cuaternión al que le falta el primer folio. Del segundo manuscrito, por conservarse un fragmento tan reducido, no podemos conocer con seguridad su número y tipología de cuadernos, aunque la edición impresa que se conserva de esta obra puede ayudarnos a establecer una hipótesis viable a este respecto.

En cuanto a la existencia de signaturas, hallamos una marca N25 en el margen superior de la segunda hoja, en la que comienza el Auto a nuestra señora del Pilar, y una marca N24 en la hoja 10, también el margen superior pero más a la izquierda, donde comienza el otro manuscrito. Esto nos hace suponer que existió un anterior manuscrito facticio que reunía ambas obras, en el que el Auto de nuestra señora de Guadalupe precedía al Auto de Nuestra Señora del Pilar. En el manuscrito 15157 quizás se colocaran en distinto orden por estar el Auto de Nuestra Señora del Pilar completo, y conservarse tan solo un fragmento del de Nuestra Señora de Guadalupe. Esta preponderancia de aquel sobre este la hallamos en el mismo título de la encuadernación moderna, en cuyo lomo solo se menciona Nuestra Señora del Pilar ${ }^{25}$.

El volumen es de tamaño $4 .^{\circ}$, y sus dimensiones son de $216 \mathrm{~mm} \times 150 \mathrm{~mm}$. Mientras que el primer texto no presenta correcciones, el segundo sí. Estas, aunque no son muchas, son muy significativas, ya que nos ayudarán a establecer la posible relación existente entre el manuscrito y la edición impresa que de tal obra conservamos.

MONTERO REGUERA, José, «Hacia una revisión de las atribuciones teatrales cervantinas: el auto de la Virgen de Guadalupe», en Actas del Tercer Congreso Internacional de la Asociación de Cervantistas, 1998. -PAZ Y MELIÁ, 2.607.

\subsection{Posible origen del manuscrito}

La primera cuestión que nos asalta cuando nos enfrentamos al manuscrito del Auto famoso de Nuestra Señora de Guadalupe es si este, como su compañero de volumen, puede ser una copia de una obra impresa anterior. Por el cotejo que hemos realizado, llegamos a la conclusión de que el Auto de Nuestra Señora del Pilar, cuyo subtítulo reza «de un yngenio», es una copia del Auto a Nuestra Señora del Pilar de Felipe Sánchez, que muy posiblemente el amanuense tomó de la edición

24. En números romanos están la primera hoja de guarda (donde aparece escrito Ntra. Sra. Del Pilar), marcada con I, y la primera hoja del manuscrito, marcado con II. A partir de la segunda hoja del manuscrito, numeración en arábigos (1-10).

25. Que un manuscrito facticio aúne sendos autos dedicados a estas advocaciones marianas es interesante por la importancia que ambas tienen para la Hispanidad. 
impresa de los Autos sacramentales y al nacimiento de Christo: con sus loas y entremeses, publicados en $1675^{26}$.

Montero Reguera, en el estudio que realiza sobre nuestra obra, apunta una serie de diferencias entre el manuscrito y la edición impresa de 1617:

El manuscrito, por ejemplo, no trae loa, a diferencia del texto impreso; el manuscrito dice figuras, mientras que el impreso dice interlocutores para referirse al Drammatis personae, que, por otra parte, sufre también alguna alteración en el orden en que aparecen; y, también ciertas variantes textuales que ejemplificaré con las acotaciones:

$$
\text { 1. }{ }^{a} \text { acotación: }
$$

Ms.: Tocan dentro arrebate y salen los quatro moros con las espadas desnudas en las manos y Aliatarfe con la uandera.

Impreso: Tocan de dentro a rebato, y salen los quatro moros con las espadas desnudas, y Aliatarfe con la Vandera.

$$
\text { 2. }{ }^{a} \text { acotación: }
$$

Ms.: Toma el pendón y vase Benalamar (fol. 9v).

Impreso: Toma el pendón, y vase Benhalamar con él (fol. 60v).

$$
\text { 3. }{ }^{a} \text { acotación: }
$$

Ms.: Sale Benalamar con Rosimonda dándola (f.10).

Impreso: Sale Benhalamar con Rosimunda pressa, amenazándola.

$$
\text { 4. }{ }^{a} \text { acotación: }
$$

Ms.: banse y quedan Benalamar y Rosimunda.

Impreso: Vanse, y quedan solo Benhalamar y Rosimunda.

$$
\text { 5. acotación: }
$$

Ms.: banse medio luchando y sale Alarico con la espada desnuda y debaxo del braço Nuestra Señora de Guadalupe.

Impreso: Entranse medio luchando, y sale Alarico con la espada, y debaxo del braço a Nuestra Señora27.

Sin embargo, la diferencia más significativa y la que nos permite afirmar con seguridad que el manuscrito no es una copia de la edición impresa de 1617, y que es anterior a dicha edición, es que en él hay un verso que no aparece en el texto impreso; concretamente, el verso número 143, perteneciente a un monólogo de Alarico. Para este monólogo el autor se sirve de la silva, modalidad métrica que, como es sabido, consiste en una serie ilimitada de versos que alterna heptasílabos y endecasílabos con rima a gusto del poeta. Por este motivo nadie ha echado de menos la falta de este verso. Es más, nosotros hemos analizado la métrica del mo- 
nólogo, por si en su libertad el autor hubiese querido seguir un mismo patrón en la construcción de la silva, pero no es asî28.

Además, es precisamente en esta intervención de Alarico donde se cercena el manuscrito. 150 es el número exacto de versos que se conservan, más el listado de «figuras» que intervienen en la obra. Después de un riguroso cotejo, hemos comprobado también que, exceptuando el verso número 143, todos los demás coinciden con los del texto editado. Aparte de las diferencias en las acotaciones vistas más arriba, tan solo encontramos variantes en algunas grafías, pero nada que afecte en ningún modo al fondo ni a la forma de la composición.

Una de las características del manuscrito que llaman a simple vista la atención es que tiene una serie de correcciones hechas con una tinta más oscura a la empleada en su redacción original. A continuación, analizaremos aquellas correcciones que nos han parecido de mayor interés.

El primer caso curioso a este respecto lo encontramos en el verso 12. Este en su primera redacción tenía dos variantes posibles, una escrita encima de la otra: «y la luz de ese / su sol turbada y sola». La mano correctora tachó esse. Tres versos más adelante, en el 15, hallamos que el corrector tachó la conjunción que nosotros notamos aquí entre paréntesis: «fuiste corona [y] gloria y ornamento». El verso 49 es también muy significativo. El autor escribió: «que no auras palmo de mi», y la corrección dice: «que non auras parte en mi». Más adelante, en el verso 71, encontramos lo siguiente. En la primera redacción se escribió: «tan satisfecho se siente», y en la corrección leemos «quan satisfecho se siente». En el 75 hay una corrección también bastante curiosa, con la que parece se quiere dar un toque arcaizante al texto original. El verso dice: «porque el que es honbre de pró», y la corrección «porque el que es home de pró». El verso 88 tiene otra corrección bastante llamativa. En el original se leía «la morisca la atropella», y la corrección dice «la morisma la atropella». Del verso 97 está corregida la palabra en cursiva, cuya versión primera no logramos descifrar con claridad: «no digas tales sandeces».

De las variantes expuestas hasta aquí, la edición impresa, curiosamente, siempre opta por la corregida. Una variante digna de comentar es la del verso 100, ya que la edición impresa en este caso no mantiene ni la versión original ni la corregida. El verso primitivo decía «si a los muros andaluces» y la corrección «si a los moros andaluces». Sin embargo, en el impreso el verso es: «si a los nobles andaluces».

En los versos 124 y 125 hay otra corrección, en este caso para solventar un problema surgido por la falta de atención del amanuense. Los versos originales dicen: «fuistes a españa por patrona / patrona amada». El verso correcto, que es el que luego se imprime, debería ser «fuistes a España por patrona amada», un endecasílabo perfecto, ya que si dejamos los dos versos tal cual están, ninguno de ellos cumpliría las reglas métricas que les son pertinentes en este pasaje de la obra (forman parte de una silva). Por ello la mano correctora tacha el «por españa» del 
primer verso y añade delante del segundo la preposición «por». De esta manera el endecasílabo ya sí es perfecto en su estructura.

\section{CONCLUSIONES}

Todo lo hasta aquí mencionado nos hace preguntarnos cuál es la relación de filiación existente entre el manuscrito y la edición impresa de 1617. Si bien es cierto que, como mencionamos, el testimonio impreso mantiene todas las correcciones que se hicieron en el manuscrito, también lo es que la edición impresa tiene algunas otras variantes que la alejan de este, como podrían ser la sustitución de figuras por son interlocutores. Una clave fundamental al respecto nos la da la variante del verso 100, que no mantiene la primitiva versión del manuscrito, ni tampoco la corregida. Esta serie de diferencias nos hacen suponer que la edición impresa de 1617 no fue tomada directamente de ese manuscrito.

Hemos de pensar al respecto que, aunque no se hayan conservado testimonios de las mismas, existieron dos ediciones impresas más de la obra, una de 1605 y otra de 1615, que pudieron perfectamente incluir variantes o correcciones sobre ese manuscrito -ya corregido - que se fueron sucediendo a lo largo de la tradición impresa. El quid de la cuestión sería averiguar si el manuscrito fue la copia en la que se basó la edición impresa de 1605, o bien es una copia de esta sobre la que se hicieron correcciones que se plasmarían en la edición de 1615.

A todo esto, debemos añadir que la licencia es de 1598, por lo que tuvo que existir un texto de esta obra perteneciente a este año o algo anterior a él. ¿Puede ser el manuscrito la versión de este texto? Podría serlo. Si nos aferramos a la idea de Sánchez Arjona de que el Auto o Comedia de la Soberana Virgen de Guadalupe fue escrito y representado en Sevilla en 1594, podríamos establecer la hipótesis de que el autor de la obra, una vez representada, decidiera darla a la imprenta. Para ello recuperó el manuscrito en limpio que presentó a la justa literaria celebrada en la capital hispalense con motivo de la celebración del Corpus (de ahí el primitivo título de «auto» y no «comedia»), lo corrigió y posteriormente lo llevó a la imprenta. A lo largo de su transmisión editorial el primitivo texto del manuscrito sufrió esas variaciones que encontramos en la edición de 1617. Si optamos por esta hipótesis, para nada descabellada desde nuestro punto de vista, el manuscrito podría ser bien un autógrafo o un apógrafo cuyas correcciones, en cualquiera de los dos casos, fueron al menos supervisadas (si no realizadas) por el autor de la obra. 


\section{BiBLIOGRAFÍA}

\section{Fuentes primarias}

Madrid, Biblioteca Nacional de España, Ms. 15157, Autos sacramentales [manuscrito], Paz, Julián, Catálogo de autos sacramentales, historiales y alegóricos por don Jenaro Alenda, BRAE, IV, 1919, p. 771.

Bartolomé Gómez de Pastrana (ed.), Comedia de la Soberana Virgen de Guadalupe y sus Milagros y Grandezas de España, Sevilla, Gómez de Pastrana, 1617.

\section{Bibliografía referencial}

Asensio, José María, «Teatro español anterior a Lope de Vega. ¿Miguel de Cervantes?», en Comedia de la soberana Virgen de Guadalupe y de sus milagros y grandezas de España, Sevilla, Librería de Bibliófilos Andaluces, 1868, pp. V-IX.

Astrana Marín, Luis, Vida ejemplar y heroica de Miguel de Cervantes Saavedra: con mil documentos hasta ahora inéditos y numerosas ilustraciones y grabados de época, Madrid, Instituto Editorial Reus, 1958.

Avalle-Arce, Juan Bautista, «Atribuciones y supercherías», en Suma Cervantina, ed. Juan Bautista Avalle-Arce y Edward Calverley Riley, Londres, Tamesis Books Limited, 1973, pp. 399-408.

Cervantes, Miguel de, Comedia de la soberana Virgen de Guadalupe y de sus milagros y grandezas de España, ed. José María Asensio, Sevilla, Librería de Bibliófilos Andaluces, 1868.

Crémoux, Françoise, «Escenificación de un culto popular: la fortuna literaria de la Virgen de Guadalupe», en Actas del XIII Congreso de la Asociación Internacional de Hispanistas, ed. Florencio Sevilla y Carlos Alvar, Madrid, Castalia / Fundación Duques de Soria, 2000, vol. I, pp. 476-484.

Domínguez Guzmán, Aurora, La imprenta en Sevilla en el siglo XVII (1601-1650). Catálogo y análisis de su producción, Sevilla, Universidad de Sevilla, 1992.

Eisenberg, Daniel, «Repaso crítico de las atribuciones cervantinas», en Nueva Revista de Filología Hispánica, 38, 1990, pp. 477-492.

Eisenberg, Daniel, ¿Qué escribió Cervantes?, Alicante, Biblioteca Virtual Miguel de Cervantes, 2003.

Menéndez Pelayo, Marcelino, Estudios y discursos de crítica histórica y literaria. Estudios generales. Edad Media influencias semíticas. Cervantismo, Madrid, Consejo Superior de Investigaciones Científicas, 1942.

Montero Reguera, José, «La obra literaria de Miguel de Cervantes (Ensayo de un catálogo)», en Cervantes, Madrid, Centro de Estudios Cervantinos, 1995, pp. 43-74. 
Montero Reguera, José, «Hacia una revisión de las atribuciones teatrales cervantinas: el auto de la Virgen de Guadalupe», en Actas del Tercer Congreso Internacional de la Asociación de Cervantistas, ed. Antonio Pablo Bernat Vistarini, Palma, Universitat de les Illes Balears, 1998, pp. 611-617.

Ortega Morejón, José María, «Primeros apuntes. La Comedia de la Soberana Virgen de Guadalupe y sus milagros y grandezas de España», en Apuntes para dos obras relacionadas con Cervantes con algunos documentos inéditos, Madrid, Tipografía de la «Revista de Archivos, Bibliotecas y Museos», 1915.

Palau y Dulcet, Antonio, Manual del librero hispano-americano: bibliografía general española e hispano-americana desde la invención de la imprenta hasta nuestros tiempos con el valor comercial de los impresos, Barcelona / Oxford, Librería Anticuaria de A. Palau, 1948-1977.

Paz y Meliá, Antonio, Catálogo de las piezas de teatro que se conservan en el Departamento de Manuscritos de la Biblioteca Nacional, Madrid, Patronato de la Biblioteca Nacional, 1934.

Sánchez Arjona, José, "Auto de La Virgen de Guadalupe, atribuido a Cervantes», en Noticias referentes a los anales del teatro en Sevilla desde Lope de Rueda hasta fines del siglo XVII, Sevilla, Imp. de E. Rasco, 1898, pp. 86-90.

Simón Díaz, José, Bibliografía de la Literatura Hispánica, Madrid, Consejo Superior de Investigaciones Científicas, 1992. 